\title{
Inklusive Exklusion durch Religion in Indien - hindu-nationalistische Tendenzen als Alarmsignal für die Weltgemeinschaft am Beispiel der National Education Policy ${ }^{1}$
}

\begin{abstract}
Zusammenfassung
Mit dem Entwurf National Education Policy von 2019 werden bildungspolitische Rahmenvorgaben für ganz Indien vorgelegt. Diese läuten einen Paradigmenwechsel der nach eigenem Bekunden größten Demokratie der Welt ein. Ideologische Argumentationen der hindu-nationalistischen Regierungspartei stellen die seit der Unabhängigkeit des Subkontinents säkularplurale Demokratie in Frage. Verfassungsmäßig garantierte Einheit in Vielfalt soll durch inkludierenden Hindu-Nationalismus ersetzt werden. Dadurch werden jene ausgeschlossen, die dieser Ideologie nicht folgen wollen. Welthistorisch implizierte Höherwertigkeit wird mit zivilisatorischen Anfängen im Indus-Tal begründet. Der Beitrag ordnet exemplarische Aspekte des Themas systematisch für aufgeklärte Weltbürger/-innen in- und außerhalb Indiens, die sich ihrer historischen Verantwortung bewusst sind.
\end{abstract}

Schlüsselworte: Indische Bildungspolitik, Hindu-Nationalismus, Hindutva, Einheit in Vielfalt

\begin{abstract}
The draft of the National Education Policy of 2019 presents an educational policy framework for whole India. This opens up a paradigm shift for the biggest democracy of the world, according to own description. The Hindu nationalist ruling party's ideological arguments question the secular-plural democracy that has existed since the subcontinent gained independence. Unity in diversity guaranteed by the constitution shall be replaced by inclusive Hindu nationalism. This excludes those who do not want to follow this ideology. The implied worldhistorical superiority is based on civilizing roots of the Indus Valley. The article sorts out exemplary aspects of the topic systematically for enlightened global citizens in and outside India who are aware of their historical responsibility.
\end{abstract}

Keywords: Indian Education Policy, Hindu Nationalism, Hindutva, Unity in Diversity

\section{Statement mit Sprengkraft}

„Wir Inder haben 330 Millionen Götter, sie nur einen. Also haben wir Inder 330 Millionen+ 1 Götter. Und wenn sie sich an unsere Gesetze halten, können sie bleiben. Wenn nicht, können sie ja nach Pakistan oder Bangladesch auswandern. Falls sie die Bevölkerungsmehrheit übernehmen, können wir Hindus nirgendwohin. Bis sie kamen, haben wir Inder immer in friedlicher Harmonie gelebt. Heute sollten wir allen die besondere globale indische Geschichte seit der Antike erzählen“.

Diese spezifische Weltsicht eines indischen Kollegen verdeutlicht, wie Indien sich als hinduistisch-identitäre Nation gegenüber anderen abgrenzen möchte. Hindus (ca. 80 \% der Bevölkerung) werden als Inder trotz großer Unterschiedlichkeit vermeintlich monolithischen Muslimen (ca. $14 \%$ ) als Inder zweiter Klasse gegenübergestellt. Dahinter liegt der machtvolle hindunationalistische Traum mit vermeintlich indoarisch antiker Zivilisation als wachsender „Griff der Hindu-Nationalisten " (Jaffrelot, 2019). Nach einer historischsystematischen Skizze werden an einigen Punkten des Entwurfs der National Education Policy (MHRD, 2019) Bezüge zur Hindutva-Ideologie der auf Bundesebene regierenden BJP (Bharatiya Janata Party/Indische Volkspartei) aufgezeigt.

\section{Inklusiv-exklusive Hindutva}

Je komplexer die Welt wird, desto mehr scheinen Menschen einfache Erklärungen zu brauchen. Diese weltweite Tendenz dürfte eine Begründung für den Aufstieg von Politikern wie Trump, Erdogan, Bolsonaro, Putin, Orban, Duterte oder Modi sein. Indus mit Hindu gleichzusetzen, ist eine ideologische Umarmungsstrategie: Inklusive Exklusion wird zum rückschrittlichen Fortschritt, indem die reichhaltige indische Geschichte und plural-säkulare Nation hindu-nationalistisch umgedeutet wird. Andersdenkende haben in einem solchen Weltbild nur dann Platz, wenn sie sich den gesetzten Spielregeln unterordnen. Partizipation ist eine zugestandene Möglichkeit der semantisch Herrschenden. 
Hinduismus als Religion einer säkular-pluralen Nation und Hindu-Nationalismus als politisches Programm stehen diametral zueinander. Indien war seit jeher ein Vielvölkerland mit verschiedenen Weltanschauungen, das Rabindranath Tagore (1861-1941) als Unity in diversity beschrieb. Der Subkontinent hat gut daran getan, vielfältige Ethnien, Religionen und Sprachen in irgendeiner Form mehr oder weniger friedlicher Koexistenz zu respektieren. Trotz Konfliktpotenzial v.a. bei sozialen Fragen konnte durch gewaltfreien Widerstand unter Führung von Mohandas K. Gandhi die Unabhängigkeit von britischer Herrschaft erlangt werden; eine weltweite Motivation für unzählige zivilgesellschaftliche Bewegungen! Dieser Erfolg war nicht ohne Paradoxien zu haben (Lang-Wojtasik, 2019). Indien ist gut 70 Jahre nach seiner Unabhängigkeit dabei, bekannte Probleme eher zu verfestigen als nach konstruktiven Lösungen gemäß eigener Verfassung zu suchen (Datta, 2017).

Die Religion des Hinduismus erscheint für europäisch sozialisierte Wahrnehmungen als kaum überschau- und verstehbar. Während der britischen Kolonialzeit war dies häufig der Ausgangspunkt dafür, seit Jahrhunderten gewachsene und ein Gemeinwesen aufrechterhaltende Traditionen aus Sicht der Herrschenden als minderwertig zu betrachten. Seit dem 19. Jahrhundert gab es mehrere Hindu-Reformbewegungen, u. a. um das Kastenwesen, Witwenverbrennungen, Benachteiligungen von Frauen oder Kinderheirat abzuschaffen und Polytheismus zu überwinden. Eine Richtung war die Brahmo Samaj (Brahma-Vereinigung), die 1828 in Kolkata von Raja Rammohan Roy (1772-1833) gegründet wurde. Sie war liberal, erkannte alle Religionen als gleichwertig an, ohne eine bewertende Einschränkung göttlicher Erscheinungen vorzunehmen, da alle Wege zum Göttlichen führen. Die Gründerväter betonten auch Gemeinsamkeiten mit dem Christentum und Islam. Die andere Richtung, der erzkonservative Arya-Samaj (Arier-Vereinigung), wurde 1825 in Bombay von Mula Sankara (1824-1883) gegründet, der sich Swami Dayananda Saraswati nannte. Dieser Zusammenschluss lehnte ebenfalls den Polytheismus ab, beanspruchte allerdings Einmaligkeit und Unfehlbarkeit der vedischen Tradition. Andere Religionen waren inexistent. Anti-christliche und -islamische Propaganda gehörte zum Programm (Stutley, 1994, S. 183-189). Eine weitere Radikalisierung erfuhr dieser vedischbrahmanische Hinduismus 1915 durch die Akhil Bharatiya Hindu Mahasabha (gesamtindische Hindu-Großversammlung). Der für sie einigende Begriff Hindutva geht auf ein Pamphlet des radikalen Nationalisten Vinayak D. Savarkar (1883-1966) in den 1920er Jahren (Wolf, 2017) zurück. Zu seinen Gefolgsleuten gehörte auch Nathuram Godse, der Mörder Mohandas K. Gandhis. Die erhoffte Solidarität der Hindus erfolgte durch eine Umdeutung der Bharat Mata (Mutter Indien) als „,heiligem Land“ der Hindus (Imhasly, 2015, S. 199f.; Rothermund, 2008, S. 40 f.). Der Arya Samaj gilt als Vorbild des 1925 gegründeten RSS (Rashtriya Swayamsevak Sangh/Nationales Freiwilligenkorps), einem hinduistisch-faschistoiden Netzwerk, das von 1948 bis 1949 im Zusammenhang mit der Ermordung Gandhis verboten war. Zusammen mit dem VHP (Vishva Hindu Parishad/,Welthindurat") ist der RSS international v.a. durch die Zerstörung der Babri-Moschee in Ayodhya (1992) oder durch die Gujarat-Pogrome gegen Muslime (2002) in Erschei- nung getreten. Neben paramilitärischen Übungen fördert er die Verbreitung des Yoga als politischem Programm im Gewand hindu-nationalistischer Harmonie und Völkerverständigung bis hin zur Einführung des UN-Yoga-Tages (ab 21. Juni 2015) in der ersten Amtszeit von Narendra Modi (Ross, 2018). Politischer Arm der Hindutva-Netzwerke ist die 1980 gegründete und seit 2019 zum dritten Mal auf Bundesebene regierende BJP.

Nationale Identität wurde einigendes Band v.a. für die Unabhängigkeit von der britischen Kolonialherrschaft. Die Mütter und Väter der indischen Verfassung schufen ein Grundlagenwerk plural-säkularer Demokratie, in dem sich alle Inder/-innen weitgehend wiederfinden konnten. In der Tradition des tiefgläubigen und alle Religionen respektierenden Hindus Gandhi als Vater der Nation gelten grundlegende Wertorientierungen: Verfassungspatriotismus und Anerkennung geteilter Vielfalt ohne Höherwertigkeit; Bekenntnis verfassungsgemäßer Bedeutsamkeit lokaler, regionaler, länder- und nationaler Entscheidungen; dynamische Entwicklung von Nation, Staat, Religion und Führungspositionen; aufrichtig beschämter Umgang mit Verfehlungen oder Verbrechen im Namen der Nation; Bereitschaft gegenseitigen kulturellen Lernens als Basis des Zusammenlebens (Guha, 2019). Die hindu-nationalistischen Umgestaltungen durch BJP-Regierungen - zunächst 1998 bis 2004 unter Atal B. Vajpayee und seit 2014 unter Narendra Modi - sind daran gemessen Verfassungsbrüche.

Der selbstkritische Weltbürger Gandhi begriff die gewaltfreie Überwindung unterdrückerischer Kolonialherrschaft als universelle Blaupause. Auch wenn er von einem Indien der autonomen Dorfrepubliken ausging, von dem sich Regierungen ab der Unabhängigkeit immer mehr verabschiedeten, bleiben die historischen Errungenschaften der plural-säkularen Nation ein motivierendes Angebot für die Weltgemeinschaft. Hindutva-Ideologie steht für phantasiereich konstruierte Reduktionen vermeintlich antiker Universalitätswurzeln, die in totalitärer Weltherrschaft enden können.

Mit der indischen Verfassung wurden einer hierarchisch-stratifikatorischen Gesellschaft inklusiv-ausgleichende Zielsetzungen an die Seite gestellt. Häufig intersektional verschränkte Diskriminierungen aufgrund von Kaste, Klasse, Geschlecht, Ethnie, Religion etc. wurden als überwindbar gedacht. Die würdevolle Wertschätzung von Sprachenvielfalt und historischer Multiperspektivität sind selbstverständliches Gut. Demgegenüber setzt hindu-nationalistische Identität auf monolithisch-ausgrenzende Reinheit indoarisch verwurzelter antiker Tradition. Hindi und Sanskrit werden zu übergeordneten Sprachen erklärt, das Kastenwesen als Teil göttlicher Ordnung begriffen und selektiver Geschichtsblick zur Grundlage des Denkens erkoren.

Was religiös unmöglich ist, wird in der Hindutva-Ideologie politisch-fundamentalistisch passend gemacht: Buddhismus, Jainismus und Sikhismus werden inklusiv hinduisiert, ohne historische Fakten oder dahinterliegende Komplexität religiöser Entwicklungen zu beachten. Partizipative Eigenständigkeit wird ausgeblendet. Das gilt noch stärker für Islam oder Christentum und letztlich alle anderen in Indien bedeutsamen Religionen wie Judentum, Parsismus, Bahai etc. Inklusive Exklusion wird auch für untere soziale Gruppen der hinduistischen Kastenordnung vollzogen. Damit werden auch verfassungsgemäße Sonderrechte für Adivasi (indische Indige- 
ne), Dalits („Zertretene“ als Eigenbegriff der „Unberührbaren“) oder Other Backward Castes (OBC) in Frage gestellt (z.B. Quotierungen öffentlicher Stellen und Studienplätze). Hindutva-Kommunalismus gipfelt als Anti-Säkularismus in der Frage, wer als Staatsbürger/-in betrachtet wird. Daraus ergibt sich eine Konfliktlinie, bei der es auch um den Ende 2019 verabschiedeten Citizenship Amendment Act oder die Entwicklungen in Kashmir und Assam geht - mit massiven Protesten von Menschen, die sich um die plural-säkulare demokratische Verfassung sorgen (Daum, 2020; Naravane, 2019).

\section{National Education Policy (NEP) als Weg- bereiterin neoliberaler Hindutva}

Gut 50 Prozent der indischen Bevölkerung sind unter 25 Jahre alt (MHRD, 2019). Über bildungspolitische Rahmenvorgaben kann ein hindu-nationalistischer Staat vorangebracht werden. Der umstrittene NEP-Draft (486 Seiten) sowie der Komitee-Bericht (217 Seiten) zu ihrer Entwicklung (MHRD, 2016) schaffen noch mehr: Der seit der Unabhängigkeit gesetzte Fokus sozialer Gerechtigkeit in Bildungsinstitutionen wird zugunsten einer ökonomisierten Hindutva umgangen (Mishra, 2019; Prasad, 2019). Frei nach dem Motto: Auf in das 21. Jahrhundert mit einer Synthese aus neoliberaler Zukunftsmusik und Verankerung in vermeintlicher Tradition eines einheitlichen hindu-nationalistischen Reiches, das seit Jahrtausenden inklusiv-friedliebend zur universalen Entwicklung der Menschheit beigetragen habe (MHRD, 2016, S. 11; 169; 2019, S. 25 f.; 235). Dazu wird das demokratisch-säkulare Grundprinzip einer Wertschätzung gleichwürdiger Vielfalt auf dem Altar durchökonomisierter hindu-nationalistischer Identität geopfert.

Indiens Bildungswesen mit aktuell 29 Bundesstaaten und sieben zentral regierten Territorien ist komplex und geschichtsträchtig. Es war nie monolithisch national oder folgte einer direkten Linie von indischer Antike bis heute, mit der universale Harmonie und Völkerverständigung vorbereitet worden wäre (Lang-Wojtasik, 2013). Im NEP-Draft wird ein „India centred education system” angestrebt. Die Nation soll nachhaltig als faire und dynamische Wissensgesellschaft umgebaut und Bildungsqualität für alle angeboten werden (MHRD, 2019, S. 41). Unklar bleibt, unter welchen Vorzeichen dies realisiert werden soll. Voraussetzung dafür wären Rahmenbedingungen, in denen Menschen Zugang zu Bildung bekommen und auch anschlussfähiges Wissen für ein demokratisches Indien mit Weltbedeutung erlangen können. Hinter „alle“ verbergen sich vermutlich all jene, die sich der Hindutva-Ideologie unterordnen wollen.

\section{Zentralisierte Hindu-Nationalisierung}

Die Macht über den Bildungsbereich soll im direkten Umfeld des Premierministers angesiedelt werden (Prasad, 2019). Er soll der dazu gegründeten Rashtriya Shiksha Aayog (RSA/Nationale Bildungskommission) unmittelbar vorstehen. Die Stellvertretung soll beim Bundesbildungsminister liegen. Die 20 bis 30 Mitglieder der RSA sind Bundesminister/-innen, „whose ministries impact education directly (e. g. health, woman and child development, finance)“ (MHRD, 2019, S. 392) sowie einige der Regierungschefs auf Ebene der Bundesstaaten. Damit soll die bisherige Kultusautonomie der Länder weitgehend zentralisiert werden (ebd., S. 391 ff.). Bildung zur Chefsache zu machen, bietet reichhaltige Chancen, einen gesellschaftlichen $\mathrm{Zu}$ kunftsbereich im Hindutva-Sinne umzubauen, der im NEPDraft geschickt mit neo-liberaler Ökonomisierung verbunden wird. Umschreibungen von Unterrichtswerken durch Sanskritisierung und anti-islamische Tendenzen, führten in der ersten (Thapar, 2004) und zweiten Amtszeit der BJP zu massiven Protesten (Buck, 2016). Willkürliche Umdeutungen indischer Geschichte in Unterrichtswerken legten eine hindu-nationalistische Sicht auf staatsbürgerliche Rechte und Pflichten zugrunde. Überhöhtes Selbst wurde zum Maß kommunalistisch-abgegrenzter Anderer auf allen Bildungsebenen (Thapar, 2004).

Die geplante Umsetzung einer 5+3+3+4-Struktur allgemeiner Schulbildung in ganz Indien bedeutet eine Überwindung des 10+2-Systems. Was wie ein Befreiungsschlag uneiniger indischer Bildungspolitik seit der Unabhängigkeit wirkt, ist letztlich ein massiver Eingriff in die Kultushoheit der Länder. Geplant sind fünf Jahre Fundamentalstufe (drei Jahre PrePrimary und zwei Jahre Primary), drei Jahre Vorbereitungsstufe (Latter Primary), drei Jahre Mittelstufe (Upper Primary) und vierjährige Oberstufe (Secondary) (ebd., S. 73 ff.). Die Orientierung an einem vierjährigen universitär organisierten B.Ed. für alle Formen der Lehrendenbildung umfasst eine Kombination aus fach-, bildungswissenschaftlichen sowie praktischen Trainingsanteilen und soll für ganz Indien gelten (MHRD, 2019, S. 134). Die Gleichwertigkeit der Lehrendenbildung mit anderen vierjährigen BA-Abschlüssen und Ermöglichung weiterführender Masterstudien in anderen Disziplinen oder im pädagogischen Bereich (ebd., S. 135) schafft internationale Anschlussfähigkeit und Vergleichbarkeit über innerindische Ländergrenzen hinweg - zum Preis direkter Steuerung aus der Regierungszentrale durch die erwähnte RSA. Die Berücksichtigung neuester Erkenntnisse von Bildung und Pädagogik suggeriert weltbürgerliche Offenheit. Allerdings muss dies in einem hindu-nationalistischen Verständnis der „Indian values, ethos, knowledge and traditions“ (ebd., S. 283) verankert werden, für die eine universale Weltverankerung im Indus-Tal angenommen wird. Leidenschaftlich-engagierte Lehrkräfte gelten als zentrale Transformationsakteure. Ihre Vorbereitung, Orientierung und Ausbildung müsse in höherer Bildung platziert werden (MHRD, 2019, S. 31). Dies wird mit vedischer Guru-Education als der (!) indischen Bildungstradition begründet (MHRD, 2016, S. 168). Lehrer seien im antiken Indien die am meisten respektierten Gesellschaftsmitglieder gewesen (MHRD, 2019, S. 113). Gleichwohl: Es gab damals kein allgemeinbildendes Schulsystem. Die Gurukul-Tradition war ausschließlich brahmanischen Jungen vorbehalten und ist Teil eines seit Jahrtausenden existierenden Exklusionssystems, die im NEP-Draft als Teil „religiöser Schulen“ (MHRD, 2019, S. 71) gepriesen wird (Prasad, 2019, S. 6).

\section{Inklusiv-höherwertige Geschichtsschreibung}

Alle Ausführungen des NEP-Draft sind redundant von der Idee durchwirkt, das glorreiche antik-indische Kulturerbe von den Ursprüngen im Indus-Tal bis heute für die Welt hervorzuheben. Unter der Überschrift „Drawing from India's heritage“ werden z.B. ganzheitliche Bildungsüberlegungen betont, die 
ihren Ausgangspunkt im antiken Indien hätten (MHRD, 2019, S. 25). Die Umdeutung von Swami Vivekanandas (1863-1902) Programm einer „kulturellen Renaissance“ des Hinduismus (Ross, 2018) ist eine Anmaßung. Denn Vivekananda stand - wie Gandhi - für religiöse Gleichwürdigkeit und universelle Akzeptanz der Geschwister auf der Suche nach göttlicher Wahrheit mit unterschiedlichen Wegen. Dies unterstrich er sinngemäß in seiner weltweit beachteten Rede auf dem ,Parlament der Weltreligionen' in Chicago 1893. Exklusive Höherwertigkeit oder Fanatismus lehnte er entschieden ab (Schlensog, 2006, S. 377 ff.). Es ist irreführend, kolonial geprägte Wissenschaft durch den wissenschaftlichen Charakter des Hinduismus ersetzen zu wollen. Gleichwohl gab es in der indischen Geschichte vielfältige wissenschaftliche Beiträge, die im NEPDraft Erwähnung finden (MHRD, 2019, S. 26). Dabei geht die Balance zwischen belegbarer Geschichte als wissenschaftlichem Beitrag und science fiction etwas verloren. Mit den Ausführungen im NEP-Draft werden Hindutva-Geschichtsumdeutungen wie die Folgenden geebnet: Vedische Mythologie wird zu Grundlagen plastischer Chirurgie oder Luftfahrt zusammengereimt. Die Heilungskräfte des Kuh-Urins für Krebsbehandlungen aus der ayurvedischen Medizin werden - trotz differierender Wissenshorizonte - als Ersatz für Allopathie gepriesen. Evolutions- oder Relativitätstheorie werden historisch relativiert. Erkennbare "Scheinwissenschaftlichkeit" (Ross, 2018) degradiert die beeindruckenden und unbestrittenen wissenschaftlichen Leistungen der antiken indischen Zivilisation zu Fußnoten der Geschichte. Es ist ein Bärendienst, den die vermeintlichen ,Retter des Hinduismus'schaffen. Das bisher weltweit unbestrittene mathematische oder astronomische Werk des Gelehrten Aryabhata (ca. 5. Jh. n. Chr.) kann sich Vereinnahmungsversuchen hindufanatischer Fake News kaum entziehen. Algebra (MHRD, 2019, S. 102) als arabischer Begriff unterstreicht den Austausch zwischen mittelalterlich-neuzeitlichem Europa sowie Nahem und Mittlerem Osten bis hin nach Indien. Die Araber bezeichneten Mathematik als hindisat (indische Kunst) (Ross, 2018).

Buddhistische Beiträge - interpretiert als Hindu-Derivat - werden in ihrem friedlichen Beitrag für Südostasien gewürdigt. Indische Bildung sei stets durch kulturelle Mischung seit ersten Invasionen bis zur Ankunft der Briten bereichert worden. Sie hätte viele Einflüsse aufgesaugt und eine einzigartig-eigenständige Kultur geschaffen (MHRD, 2019, S. 26). Der Universalisierungscharakter indischer Antike in den buddhistischen Universitäten von Taxila, und Nalanda, Vikramshila oder Vallabhi mit Besuchern asiatischer Regionen sei direkt mit heutiger Internationalisierung höherer Bildung verbunden (ebd., S. 249). Klassische Sanskrit-Literatur wäre quantitativ höherwertiger gegenüber lateinischen und griechischen Schriften (ebd., S. 86).

Die Entstehung der Liberal Arts wird im NEP-Draft als Teil der indischen Antike verortet, in der ganzheitliche Aspekte menschlichen Wissens als grundlegend interdependent betrachtet wurden (MHRD, 2019, S. 29) und für alle Bildungsbereiche relevant seien. Die „Knowledge of India“ soll in Abgrenzungzu europäischem Wissen vertieft werden. Baudhayana, Virahanka, Pingala oder Aryabhata werden explizit gegenüber Pythagoras, Fibonacci oder Pascal in Stellung gebracht (ebd., S. 98). Höhere Bildung und damit auch die Lehrendenbildung wird als „Liberal Education“ der indischen Antike begriffen.
Die 64 kalas (Künste) seien vor mehr als 1400 Jahren als „firstever novels“ (ebd., S. 223) entstanden. Ihre Bedeutung sei herausragend für die kreativanalytische Förderung individueller Entwicklung, die kommende „fourth industrial revolution“ (ebd., S. 224) sowie die Bestärkung von Wissenschaft zwischen indischer Tradition und universeller Moderne. Hier muss dann selbst Steve Jobs herhalten, der den Erfolg von Macintosh-Computern v.a. mit ihrer multidisziplinären, künstlerischen wie naturwissenschaftlichen Nutzung begründe (ebd., S. 225).

\section{Inklusiv-exklusive Hindutva-Diversität}

Indien wird redundant als diverse Wiege von Traditionen, Sprachen, Dialekten, kulturellen Entwicklungen usw. gepriesen und sei die Keimzelle des kommenden Bildungssystems mit antiken Wurzeln und Weltwirkung. Offen bleiben das Maß und die Eigenständigkeit von Vielfalt, die jenseits von Hindutva-Fantasien gelebt werden darf. Indien hat je nach Perspektive mind. 22 Hauptsprachen und geschätzt mehr als 1600 gesprochene Sprachen. Der Schutz der Sprachenvielfalt ist verfassungsmäßig gewährt (Art. 343-351 \& Schedule 8). Seit spätestens Mitte der 1960er Jahre gilt die seit der Unabhängigkeit geforderte Drei-Sprachen-Politik als allgemein akzeptierte Grundlage (Mutter-/Regionalsprache + Hindi/Englisch + indische Hauptsprache/internationale Fremdsprache, die nicht zu den ersten beiden gehört) (Gupta, 2007, S. 89 f.). Damit sollte auch ein gewisser Ausgleich zwischen nord- und süd-indischen Sprachen stattfinden, also nordindisch Sprechende eine südindische Sprache und umgekehrt erlernen.

Die im NEP-Draft geforderte Unterordnung dieser Vielfalt unter Hindi und Sanskrit als leitenden Nationalsprachen - neben Englisch als Amts- und internationaler Sprache - ist eine Anmaßung. Die Amtssprache Hindi als übergreifend-höherwertige Sprache zu begreifen, war seit jeher in nicht-hindi-sprechenden Regionen v.a. Ost-Indiens, im dravidisch sprechenden Südindien oder den indigenen Regionen sehr umstritten. In einem „fun course“ (MHRD, 2019, S. 85) irgendwann zwischen der sechsten und achten Klasse sollen Schüler/-innen die v. a. in Sanskrit wurzelnde „remarkable unity" (ebd.) der meisten indischen Sprachen sowie die Grundstruktur der indigenen Sprachen kennenlernen. Ausgewählte Sätze in den vielfältigen Sprachen sollen ihnen als ,icebreaker“ für ihr ganzes Leben dienen, wenn sie Menschen aus allen Teilen des Subkontinents treffen (ebd., S. 86). Letztlich soll die indische Sprachenpolitik aus der brahmanischen Gelehrtensprache Sanskrit heraus begründet werden, die zur, modernen Sprache ' mit nationalem Einigungspotenzial erklärt wird (ebd.). Ihr werden andere klassische (nicht moderne!) Sprachen Indiens wie Pali, Prakrit und Persisch an die Seite gestellt. In diese Gruppe werden auch Sprachen einsortiert, die in der indischen Realität des 21. Jahrhunderts Verkehrssprachen von Millionen Menschen Südindiens sind: Tamil (v. a. in Tamil Nadu), Telugu (v. a. in Andra Pradesh), Kannada (v.a. in Karnataka), Malayalam (v. a. in Kerala) und Odia (v. a. in Orissa). All dies ist ein Affront gegen die plural-säkulare Verfassung.

Der zentralisierte Inklusionsanspruch im Hindutva-Gewand exkludiert all jene, die als lokal, regional oder statelevel subsumiert werden. Den Draft der NEP nur in Englisch und Hindi verfügbar zu machen, erzeugt eine explosionsförder- 
liche Lunte, die an die indische Verfassung angelegt wird (Prasad, 2019). Wenn mit Sanskrit der unterstellte Globalcharakter des Hinduismus unterstreichbar wäre; warum konzentrieren sich erlernbare Fremdsprachen der Sekundarschule nur auf Französisch, Deutsch, Spanisch, Chinesisch und Japanisch (MHRD, 2019, S. 84)? Arabisch oder afrikanische Sprachen werden ausgeblendet (Roy, 2019).

Curricular soll ganzheitliche Entwicklung in hindu-nationalistischem Sinne vorangebracht und sollen damit verbundene Fertigkeiten für das 21. Jahrhundert gefördert werden: „critical thinking, creativity, scientific temper, communication, collaboration, multilingualism, problem solving, ethics, social responsibility and digital literacy" (MHRD, 2019, S. 73). Dies wirkt auf den ersten Blick modern und international anschlussfähig. Allerdings basieren die ethisch-moralischen Prinzipien auf Sanksrit-Semantik,die hindu-nationalistische Höherwertigkeit unterstreichen soll: „seva, ahimsa, swacchata, satya, nishkam karma“ - also Dienst am anderen, Nicht-Hass, Reinheit, Wahrheit, selbstloser Dienst - zusammen mit „tolerance, honest hard work, respect for women, respect for elders, respect for all people and their inherent capabilities regardless of background, respect for environment, etc. will be inculcated in students" (MHRD, 2019, S. 96).

Das National Curricular Framework (NCF) soll überarbeitet werden und als letztlich hindu-nationaler Rahmen ,lokale Inhalte und Geschmäcker' berücksichtigen (MHRD, 2019, S. 101). Damit werden vielfältige und eigenständige Traditionen, die die demokratiegeleitete Einheit in Vielfalt seit langem ermöglichten, einer vermeintlich höherwertigen und leitenden Hindu-Nation untergeordnet. Religiöse Bildungseinrichtungen (madrasas/maktabs, gurukuls, pathshalas) müssen sich am mit dem im NEP-Draft festgeschriebenen NCF ausrichten (ebd., S. 151), der national-hinduistisch ausgerichtet ist - mit entsprechendem Konfliktpotenzial. Denn diese Direktive greift direkt in das verfassungsgemäße Recht auf Eigenständigkeit religiöser Bildungseinrichtungen ein (Art. 30).

\section{Antik-neoliberaler Angriff auf die Verfassung}

Die Grundfesten der indischen Verfassung geraten mit dem NEP-Draft massiv ins Wanken. Das beginnt damit, bereits in der Einleitung auf die Erwähnung ebendieser zu verzichten und ihre zentrale Bedeutung für die indische Demokratie zu unterminieren (Roy, 2019). Auch die im Komitee-Bericht (MHRD, 2016) bemühten Bezüge zur Wardha-Konferenz (1938) und der Nai Talim (New Education) im Sinne Gandhis sowie die in der Folge formulierten Ziele einer „indianisierten“ Bildung (ebd., S. 11) helfen nicht. Immerhin war Gandhis Bildungsvision von einem friedliebenden Indien geprägt, in dem Einheit in gleichwürdiger Vielfalt auch für Bildung entfalten werden sollte.

Fast 35 Jahre nach dem Erscheinen der letzten bildungspolitischen Rahmenvorgaben hätte der NEP-Draft ein Wurf für die nächsten Jahrzehnte mit verfassungsgemäßer Bodenerdung sein können. Allerdings bricht der Entwurf an verschiedenen Stellen mit säkulardemokratischen Traditionen seit der Unabhängigkeit, die leitend für die Vorgängerpapiere von 1968 sowie 1986/1992 unter der Ägide Congress-geführter Regierungen waren. Der Bericht der ersten Bildungs-Kommission (Kothari-Commission) Ende der 1960er Jahre hob die
Umsetzung einer lernenden Gesellschaft aus Lernenden und Lehrenden hervor, in der verschiedene Zielsetzungen einen Grundkonsens jenseits von Uniformierungen darstellten. Leitend war die Freiheit der Lernenden für eine demokratisch-säkulare Gesellschaft. Politische, ökonomische oder religiös-ideologische Einflussnahme sollte so gut wie möglich unterbunden werden (Prasad, 2019). Dieser gerechtigkeitsgeleitete Grundkonsens wird unter dem Vorzeichen leistungsorientierter Kriterien weitgehend verabschiedet, die geschickt mit hindu-nationalistischer Inklusion verbunden werden. Es wird unterstellt, dass letztlich jede und jeder jenen Platz in der Hindu-Nation findet, die seit den vedischen Ursprüngen als möglich angenommen werden.

Bisher zentrale Orientierungspunkte des Zuganges und der Gleichheit - mit gezielten Unterstützungsmöglichkeiten benachteiligter Gesellschaftsgruppen - werden v.a. durch individualisierte Qualitätsorientierung ersetzt (MHRD, 2019, S. 26), die hindu-nationalistisch gerahmt wird. Gezielte Förderung durch lange bewährte - wenn auch immer wieder kritisierte-Quotierung für gesellschaftlich benachteiligte Gruppen ist massiv unterrepräsentiert (Prasad, 2019) und geht im Vergleich zu anderen Ausführungen fast unter (MHRD, 2019, S. 148 ff.). Der mehrfach bemühte Terminus „underrepresented groups“ (z.B. MHRD, 2019, S. 66) ist eine euphemistische Bezeichnung für gesellschaftlich Exkludierte, die in säkular-pluraler Tradition besondere Aufmerksamkeit erforderten (Prasad, 2019, S. 5). Sie sollen - etwa beim Übergang in den tertiären Bildungsbereich - ,ermutigt“ werden (MHRD, 2019, S. 241).

Fördern und fordern sind eine pädagogische Mischung, die durchökonomisiert auch im neuen Indien der Hindutva-Ideologie auf „Humankapital“ setzt. Das daran anschlussfähige OECD-geprägte Modell spezifischer Kompetenz- und Output-Orientierung international vergleichender Bildungssystemvergleiche ist auch in führenden Wirtschaftsnationen umstritten (Bloem, 2016). Effektivität und Effizienz schulischer Bildung werden mit kognitiver Leistungsmessung im Sinne individueller Verdienste verbunden. Gesellschaftliche Risikogruppen bleiben ohne spezifische Förderung weltweit auf der Strecke, wenn es um intellektuelle Vorbereitungen auf Erfordernisse einer Wissensgesellschaft geht. Wie soll das Ganze in Indien funktionieren, in dem nach UNDP-Angaben fast $30 \%$ der Bevölkerung in multidimensionaler Armut leben (http://hdr.undp.org/en/countries/profiles/IND) und v.a. um das tägliche Überleben kämpfen? Die Konzentration auf „merit-based admissions“ (z.B. MHRD, 2019, S. 111) bedeutet Fordern ohne fördernden Rahmen. Neoliberale wird mit hindu-nationalistischer Ideologie verbunden.

Diskriminierung wird zu einem persönlichen Schicksal, dem man möglicherweise im nächsten Leben entfliehen kann. Der in Indien seit jeher grassierende, v.a. hinduistisch begründete Fatalismus bekommt so ein als modern interpretiertes und reaktionär hindu-nationalistisches Weltbild an die Seite gestellt. Unterdrückung wird als Gott gegebener und Teil eines kulturellen Erbes gesetzter Teufelskreis von Oben und Unten zementiert: Wie soll aber ein Kindersklave der Dalits in einem Steinbruch schulische Leistungen für gesellschaftlichen Aufstieg erbringen, wenn seine Familie seit Generationen in Schuldknechtschaft lebt? Wie soll eine jugendliche Adivasi in 
der Schule erfolgreich sein, wenn ihre Muttersprache dort nur eine untergeordnete Rolle spielt und die Unterrichtssprache erste Fremdsprache ist? Welche Möglichkeiten schulischer Bildung hat ein Kuhjunge im ländlichen Indien, der täglich den Büffel des Großgrundbesitzers hütet?

Diskriminierungen zu benennen, wird zu einer semantischen Übung ohne Konsequenzen. An grundlegenden Strukturen soll nichts geändert werden. Möglichkeiten zur Einrichtung von „Special education zones“ in benachteiligten Regionen z.B. für Adivasis (ebd., S. 141) liegt in der Hoheit der Länder. Was genau soll dort stattfinden? Der Begriff wirkt wie eine Mischung aus hingeworfenen Brotkrumen und Sonderwirtschaftszonen, mit denen massiv Landraub im Namen ökonomischer Interessen vorangebracht wird und v.a. benachteiligte Menschen in ländlichen Regionen ihrer Lebensgrundlagen beraubt werden (Müller, 2014, S. 139 ff.).

\section{Demokratische Rückbesinnung mit Fragezeichen} Trotz notwendiger Fundamentalkritik im Namen der indischen Verfassung gibt es auch Erfreuliches zu vermelden. Das Ministry of Human Ressource Development soll in Ministry of Education umbenannt werden, um Bildung und Lernen als genuinen Aufgabenbereich hervorzuheben (MHRD, 2019, S. 392). Bezüge zu internationalen Vereinbarungen - v. a. Allgemeine Erklärung der Menschenrechte (Art. 26), Delors-Bericht (ebd., S. 24 f.) oder UN-Sustainable Development Goals (Zielbereich 4) (ebd., S. 27 f.) unterstreichen das Bekenntnis zu universaler Bildungssemantik. Die Umsetzung gleicher, inklusiver und diskriminierungsfreier Bildung wäre damit die Messlatte für alle Bemühungen im weltgemeinschaftlichen Kontext (MHRD, 2019, S. 137 ff.). Gemäß Artikel 21-A der indischen Verfassung (2002) und dem „Right to Education Act" (2009) soll kostenfreie und verpflichtende Schulbildung in nahegelegenen Schulen für alle 6- bis 14-Jährigen mindestens bis zum Abschluss der Elementary School (Ende der achten Klasse) (MHRD, 2019, S. 26 f.; 2016, S. 33) realisiert werden. Dies schließt die Reintegration aller 3- bis 18-jähriger Abbrecher/-innen bis 2030 (MHRD, 2019, S. 65 ff.) ein. Frühkindliche Erziehung für alle 3- bis 6-Jährigen bis 2025 (ebd., S. 45 ff.), die kostenlose Ausgabe von Mittagessen in Vor- und Primarschulen sollen ausgebaut (ebd., S. 58) und eine Alphabetisierung für alle - bei aktuell 26.5 Mio. Analphabet/-innen (über 15-Jährige) - bis 2025 umgesetzt werden (ebd., S. 55 ff.; 373 ff.). Eine effizient-effektive Schulverwaltung sowie transparentrechtschaffene Steuerung und Implementation von Zulassungsstrukturen sollen gefördert werden (ebd., 2019, S. 73 ff.).

Nur: Wie soll das alles finanziert werden? Die absehbare Steigerung öffentlicher Bildungsausgaben auf sechs Prozent des BIP (MHRD, 2016, S. 176; 2019, S. 406) ist eine lang bekannte Forderung des unabhängigen Indien. Die Erhöhung der Gesamtausgaben für Bildung von 10 auf $20 \%$ scheint gleichwohl kaum durchsetzbar, da ein Großteil der Finanzen aus den Bundesstaaten kommen müsste. Wenn ein Teil von Philantrop/-innen oder Unternehmen beigesteuert würde (MHRD, 2019, S. 407), geriete die staatlich garantierte relative Bildungsautonomie in Gefahr. Es bestünde das Risiko ideologischer Einflussnahme (Mishra, 2019).

\section{Perspektiven}

Indiens Anspruch, die größte Demokratie der Welt zu sein, wird durch hindu-fundamentalistisch und identitär-faschistoiden Nationalismus ohne Not verspielt (Müller, 2014, S. 81 ff.). Darin liegt eine Parallele zu neurechten Bewegungen weltweit, die mithilfe kruder Geschichtsinterpretationen und konstruierter Ideologie-Phantasmen für reine Kulturen eintreten. Vergessen wird, dass Multikulturalität die Grundlage demokratischer Verfassungen und säkular-pluraler nationaler Identität sind. In diesem Sinne ist dann nicht nur interessant, was historisch überhöht, sondern auch was weggelassen wird. Eingriffe in Bildungs- und Kultuspolitik zur Verbreitung bestimmter Weltsichten sind ein historisch bewährtes Mittel fundamentalistischer Politik für ideologische Infiltration kommender Generationen.

Möglicherweise hülfe Indien eine Rückbesinnung auf die moralische Größe Gandhis. Er hatte in den 1920er Jahren in spiritueller und historischer Größe dem säkular-pluralen Indien mit auf den Weg gegeben, sich an der Überwindung sieben sozialer Sünden messen zu lassen: „Politik ohne Prinzipien, Wohlstand ohne Arbeit, Genuss ohne Gewissen, Wissen ohne Charakter, Geschäft ohne Moral, Wissenschaft ohne Menschlichkeit, Religion ohne Opfer“ (Gandhi, 1925). Ihre Gegensätze müssten die Leitlinien zukunftsfähiger Politik in Indien und darüber hinaus sein.

Indo-arische Hindutva-fanatisierte Politik weiter Teile der BJP mit historisch-universalem Anspruch ist aus deutscher Sicht mehr als bedenklich. Nationalistisch-identitäre Fantasien einer arischen Weltherrschaft mit vermeintlich sozialistischer Orientierung haben die Welt 1933-1945 in den Abgrund geführt. Das Bildungssystem war zentral, um Ideologie voranzubringen. Der indische NEP-Draft ist ein Baustein in einer längeren Reihe eines nationalistisch-hinduistischen Umbaus der säkular-pluralen indischen Republik. Universale Errungenschaften demokratisch-aufgeklärter Moderne mit reichhaltiger Geschichte, Weltanschauungen, Sprachen und Religionen werden als inklusive Umarmungspolitik ohne geduldeten Widerspruch in vermeintlich nahtloser indischer Geschichte seit der Antike exklusiv versenkt. Wie war das damals im arisch-ideologisierten Deutschland, dem christlich-jüdischen Abendland?

\section{Epilog}

„This National Education Policy envisions an education system rooted in Indian ethos that contributes directly to transforming India, that is Bharat, sustainably into an equitable and vibrant knowledge society, by providing high-quality education to all, and thereby making India a global knowledge superpower" (MHRD, 2020, S. 6). Damit ist fast alles über die 65 Seiten umfassende offiziell verabschiedete NEP gesagt. Gerade die gebetsmühlenartige Wiederholung des „vibrant“ ist ein Schlag ins Gesicht jener, die um ihr tägliches Überleben kämpfen!

Immerhin ist es gelungen, den Ursprungsentwurf um etwa ein Sechstel zu kürzen und die Erwähnung einer Konzentrierung des Bildungsbereichs beim Premierminister im Rahmen des RSA fehlt. Gleichwohl haben es manch andere Formulierungen explizit und andere Botschaften eher implizit verdeckt in die offizielle Policy geschafft. Vieles wird hinter weltoffen anmutender Terminologie verborgen. Wer kann schon etwas gegen Respekt einer "rich diversity and culture“ 
(ebd., S. 4), der Nennung von „socially disadvantaged groups“ (ebd., S. 10), der Erwähnung des „Right of Children to Free and Compulsory Education Act 2009“ (ebd., S. 4) oder die Bezeichnung von Menschen mit spezifischen (Lern-)Bedürfnissen als divyang (,göttlicher Körper') (ebd., S. 25) haben? Wer gleichzeitig weiß, wie Indien hindu-nationalistisch mit seinen Minderheiten umgeht und dass die bisher weit verbreite spezifische Förderung Benachteiligter eher zu einem Lippenbekenntnis geschmolzen ist, bekommt eine Idee davon, wie dünn das Papier ist, auf dem die politischen Absichtserklärungen formuliert wurden. Im Kern wird die neoliberale Hindutva-Agenda des NEP-Entwurfs fortgeschrieben, die stellenweise in blumigere liberal-demokratische Sprache verpackt wird. Es dominieren Sanskrit- und Hindi-Begriffe als hinduistische Rahmung, mit der das große kulturelle Erbe der Antike des Indus-Tales in seiner globalen Bedeutsamkeit bis heute unterstrichen werden soll. Der parallele Verzicht auf religiöse Vielfalt suggeriert, dass es dazwischen keine andere Geschichte gegeben haben kann. Damit wird die inklusiv-exklusive Hindutva geschickt gesetzt. Es empfiehlt sich daher, progressiv klingende Forderungen nach Multilingualismus, (ebd., S. 13), „Global Citizenship Education“ (ebd., S. 15; S. 37) oder „environment education“ (ebd., S. 23) sehr kritisch zu lesen. Immerhin werden globale Aspekte in einem Atemzug mit den holistischen Errungenschaften des antiken Indiens genannt (ebd., S. 37). Sogar „tribal knowledge“ (ebd., S. 16) soll berücksichtigt werden. Nur unter welchen Vorzeichen? Die Vielfalt indischer Sprachen, Kunst und Kultur wird in einen direkten Zusammenhang mit in- und ausländischem Tourismus gestellt (ebd., S. 53f.). Hier wäre partizipativer Perspektivenwechsel ein probates Mittel der Wahl.

The proof of the pudding is the eating! Es bleibt die Hoffnung auf die Geduld des Papiers und die historische Erinnerung, dass die letzte NEP (1986) von der Verabschiedung bis zur Überarbeitung und Implementation vier Jahre brauchte. Auch die aktuelle NEP muss in Ausführungsbestimmungen (Programme of Action) gegossen werden, deren Entwicklung kritisch-konstruktive Begleitung erfordert. Vor allem braucht es hier die Unterstützung der Bundesstaaten, die im Konsultationsprozess weitgehend übergangen wurden und die fast $80 \%$ der Bildungsausgaben tragen. An dieser Stelle motiviert dann auch die wissenschaftliche Gelassenheit eines indischen Kollegen der Delhi-University: „that what makes this document different, is essentially its claim that is is different" (Menon, 2020, S. 600).

\section{Anmerkung}

1 Die National Education Policy (NEP) wurde Ende Juli 2020 vom indischen Bundeskabinett ohne vorherige Debatte in der Lok Sahbha oder Konsultationen der Bundesstaaten verabschiedet (Nagari, 2020). Sie war und bleibt sehr umstritten (Clemens \& Vollmer, 2020). Der vorliegende Text konzentriert sich auf die Entwicklung der NEP. Eine Einschätzung des offiziellen Papiers wird in einem Epilog vorgenommen. Für kollegiale Rückmeldungen zu einer früheren Fassung zu meinem Beitrag danke ich Asit Datta.

\section{Literatur}

Bhalla, J. (2019). Draft National Education Policy 2019. Social \& Political Research Foundation. Zugriff am 29.05.2020 https://www.sprf.in/post/2019/07/26/draftnational-education-policy-2019
Bloem, S. (2016). Die PISA-Strategie der OECD. Zur Bildungspolitik eines globalen Akteurs. Weinheim u. Basel: Beltz.

Buck, K. D. (2016). Ein heiliges Buch sorgt für Unruhe. In Indien sind die Hindu-Nationalisten auf dem Vormarsch. Welt-Sichten, 58(2).

Clemens, I. \& Vollmer, T. (2020). Nationalisierungstendenzen im Bildungssystem am Beispiel Indiens und der Hindutva. Tertium Comparationis, 25(1), 28-44.

Datta, A. (2017). Indien nach 70 Jahren Unabhängigkeit. Meine Welt, 34(2), 13-15. Daum, P. (2020). Indien in der Identitätsfalle. Le Monde diplomatique, 3(20).

Gandhi, M. K. (1925/1968). Notes. Young India, 22-10-1925. In M. K. Gandhi (Hrsg.), The Collected Works of Mahatma Gandhi. Bd. 28. Ahmedabad: Navajivan, 360-365.

Guha, R. (2019). Ramachandra Guha on Rise of Hindutva \& 4 Reasons Behind "Jingoism". Zugriff am 19.05.2020 https://www.thequint.com/news/india/ramachandra -guha-on-the-rise-of-hindutva-and-4-reasons-behind-indias-jingoism

Gupta, A. (2007). Schooling in India. In A. Gupta (Hrsg.), Going to school in South Asia (66-111). Westport u. London: Greenwood .

Imhasly, B. (2015): Indien. Ein Länderporträt. Berlin: Christoph Links.

Jaffrelot, C. (2019). Indien im Griff der Hindu-Nationalisten. Le Monde diplomatique, $7(19)$.

Lang-Wojtasik, G. (2013). Bildung in Indien. In C. Adick (Hrsg.), Bildungsentwicklungen und Schulsysteme in Afrika, Asien, Lateinamerika und Karibik. Münster u. a.: Waxmann, 213-231.

Lang-Wojtasik, G. (2019). Gandhis Beitrag für eine zukunftsfähige Welt. Meine Welt, 36(2), 19-21. https://doi.org/10.2307/j.ctvpbnpz8.4

Menon, Shyam (2020). NEP 2020: Some Searching Questions. Social Change, 50(4), 599-602. https://doi.org/10.1177/0049085720958811

Mishra, A. N. (2019). Is draft National Education Policy (NEP) 2019 a declaration of privatisation? Zugriff am 26.05.2020 http://opinionpress.in/?p=1001

MHRD - Ministry of Human Resource Development (2016). National Policy on Education 2016. Report of the Committee for Evolution of the New Education Policy. Zugriff am 08.05.2020 https://planipolis.iiep.unesco.org/sites/planipolis/files/resso urces/india_report_committee_new-education-policy-2016.pdf

MHRD - Ministry of Human Resource Development (2019). Draft National Education Policy. Zugriff am 08.05.2020 https://mhrd.gov.in/sites/upload_files/ $\mathrm{mhrd} /$ files/Draft_NEP_2019_EN_Revised.pdf

MHRD - Ministry of Human Resource Development (2020). National Education Policy 2020. Zugriff am 30.11.2020 https://www.education.gov.in/sites/upload files/mhrd/files/NEP_Final_English_0.pdf

Müller, D. (2014). Indien. Die größte Demokratie der Welt? Marktmacht, Hindunationalismus, Widerstand. Berlin u. Hamburg: Assoziation A.

Nagari, A. (2020). National Education Policy not passed in Parliament, states not taken into confidence: WB minister. Hindustan Times, 01.08.2020. Zugriff am 30.11.2020 https://www.hindustantimes.com/education/national-education-policy-not-pass ed-in-parliament-states-not-taken-into-confidence-wb-minister/story-WcAyPtzte46KnOUKJCYJOJ.html

Naravane, V. (2019). Gefährliches Spiel in Kashmir. Le Monde diplomatique, 20(19). Prasad, M. (2019). NEP 2019: The devil in the detail. Frontline, 17(19). Zugriff am 24.05.2020 https://frontline.thehindu.com/cover-story/article28259123.ece

Ross, J. (2018). Spirituelle Schwerkraft. Die Zeit, 21(18), 35.

Rothermund, D. (2008). Indien. Aufstieg einer asiatischen Weltmacht. München Beck.

Roy, K. (2019). Examining the Draft National Education Policy, 2019. Economic and Political Weekly, 54(25). Zugriff am 29.05.2020 https://www.epw.in/engage/ article/examining-draft-national-education-policy-2019

Schlensog, S. (2006). Der Hinduismus. München u. Zürich: Piper.

Stutley, M. (1994). Was ist Hinduismus? Eine Einführung in die große Weltreligion. München u. Wien: Otto Wilhelm Barth.

Thapar, R. (2004). The Future of the Indian Past. Outlook. Zugriff am 18.05.2020 https://www.outlookindia.com/website/story/the-future-of-the-indian-past/223 453

Wolf, S. O. (2017). Savarkar - ein „Volksfeind“ oder Nationalheld? Dokumentation einer Debatte über das Selbstverständnis der indischen Nation. In G. Schucher \& C. Wagner (Hrsg.), Indien 2005: Politik, Wirtschaft, Gesellschaft. Hamburg: Institut für Asienkunde, 315-336.

\section{Dr. Gregor Lang-Wojtasik}

ist Professor für Pädagogik der Differenz, Pädagogische Hochschule Weingarten; er bereist Indien seit 1987. 